\title{
Determinação espectrométrica dos flavonóides das folhas de Maytenus (Celastraceae) e de Passiflora (Passifloraceae) e comparação com método CLAE-UV
}

\author{
Regina de A. O. Chabariberi, Alessandra C. S. Pozzi, \\ Maria Luiza Zeraik, Janete H. Yariwake*
}

\author{
Instituto de Química de São Carlos, Universidade de São Paulo, Caixa Postal 780, 13560-970 \\ São Carlos-SP, Brasil
}

\begin{abstract}
RESUMO: Este trabalho apresenta uma modificação dos procedimentos descritos nas Farmacopéias Francesa e Européia para a análise de flavonoides de Passiflora incarnata L., Passifloraceae, por espectrometria UV-Visível e propõe a sua aplicação na determinação dos flavonoides totais das folhas da espinheira-santa (Maytenus aquifolium Mart. e Maytenus ilicifolia (Schrad.) Planch., Celastraceae) e do maracujá (Passiflora edulis Sims. e Passiflora alata Curtis, Passifloraceae). Os resultados obtidos por espectrometria no UV-Visível foram comparados aos obtidos por cromatografia líquida de alta eficiência (CLAE-UV), encontrando-se resultados estatisticamente similares entre os métodos espectrométrico modificado da Farmacopéia Francesa e CLAE-UV.
\end{abstract}

Unitermos: flavonoides, espectrometria UV-Visível, fitoterápicos, Maytenus spp., Passiflora spp.

\begin{abstract}
Spectrometric determination of flavonoids from Maytenus (Celastraceae) and Passiflora (Passifloraceae) leaves and comparison with an HPLC-UV method". This paper reports on a modification of the spectrometric procedures originally described in the French and European Pharmacopoeia for the analysis of Passiflora incarnata L. (Passifloraceae) flavonoids, proposing its application in the determination of total flavonoids from "espinheira-santa" (Maytenus aquifolium Mart. and Maytenus ilicifolia (Schrad.) Planch., Celastraceae) and "maracujá" leaves (Passiflora edulis Sims and Passiflora alata Curtis, Passifloraceae). A comparison was made of the results obtained by the spectrometric procedure with those obtained by high performance liquid chromatography (HPLC-UV), which demonstrated complete compatibility between the modified French Pharmacopoeia (spectrometric) and HPLC-UV methods.
\end{abstract}

Keywords: flavonoids, phytomedicines, UV-visible spectrometry, Maytenus spp., Passiflora spp.

\section{INTRODUÇÃO}

O controle de qualidade de fitoterápicos é imprescindível, pois pode ocorrer problemas sérios devido à comercialização de fitoterápicos de má qualidade ou adulterados (Balmé, 1982). A padronização química de medicamentos fitoterápicos e a garantia da sua eficácia e segurança requerem métodos analíticos adequados para a deteç̧ão e quantificação dos princípios ativos (ou dos "marcadores") e de substâncias potencialmente tóxicas (Carvalho et al., 2008). Assim, o Grupo de Análise Fitoquímica do IQSC-USP tem se dedicado ao estudo sistemático de algumas plantas medicinais brasileiras, dentre as quais Maytenus aquifolium Mart. e Maytenus ilicifolia (Schrad.) Planch. (Celastraceae), conhecidas popularmente como espinheira-santa (Diagone, 2003; Tiberti et al., 2007) e de várias espécies de Passiflora (Passifloraceae) conhecidas como "maracujá" (Pereira et al., 2000; Pereira et al., 2004; Pereira et al., 2005).
Maytenus aquifolium e Maytenus ilicifolia são plantas medicinais de uso popular tradicional. Originárias do sul do Brasil, são também encontradas no Chile, Paraguai, Uruguai e Argentina (Scheffer, 2004). A quarta edição da Farmacopéia Brasileira (2002) registra a espécie Maytenus ilicifolia, e estudos farmacológicos comprovaram a sua atividade contra úlceras gástricas e gastrites em animais de laboratório (Souza-Formigone et al., 1991) e em seres humanos (Carlini, 1988). Existem relatos do uso das espécies de espinheira-santa para outras doenças, Mariot \& Barbieri (2007) discutem a ação farmacológica destas espécies em sua revisão da literatura.

As folhas de maracujá são empregadas por sua ação calmante em casos de insônia e irritabilidade; estas propriedades são atribuída às espécies Passiflora incarnata L. e Passiflora alata Curtis, Passifloraceae, (Oga et al., 1984). Porém, constatou-se que no comércio brasileiro freqüentemente há substituição de Passiflora alata por outras espécies, dentre as quais $P$. edulis fo. flavicarpa $\mathrm{O}$. 
Deg.; este fato significa fraude, pois não existem trabalhos que comprovem a validade do uso desta última como hipnótico/sedativo (Pereira et al., 2000). Passiflora edulis Sims, conhecida vulgarmente por maracujá amarelo, é a matéria-prima para o suco de maracujá industrializado no Brasil (Mercadante et al., 1988).

Técnicas cromatográficas instrumentais, tais como a cromatografia líquida de alta eficiência (CLAE) e a cromatografia de camada delgada de alta eficiência (CCDAE; em inglês, HPTLC) demonstraram ser importantes ferramentas para a identificação de material vegetal de espécies de Passiflora, através do uso de flavonoides como marcadores (Pereira et al., 2004). A CLAE também demonstrou ser uma opção eficiente para a análise quantitativa de flavonoides de Passiflora (Pereira et al., 2004) e de Maytenus (Diagone, 2003; Yariwake et al., 2005). No entanto, documentos oficiais como as Farmacopéias Francesa, Européia e Brasileira ainda adotam a espectrometria UV-Visível como método de escolha para a análise quantitativa de flavonoides em material vegetal. Para o trabalho de rotina, as técnicas espectrométricas apresentam importantes vantagens em comparação com a CLAE, tais como o menor custo e a simplicidade operacional.

Dentro desse contexto, este trabalho apresenta uma modificação do método originalmente descrito nas Farmacopéias Francesa e Européia para a determinação espectrométrica de flavonoides em Passiflora incarnata (European Pharmacopoeia, 2000; Pharmacopée Française, 1992) com o objetivo de desenvolver uma metodologia adequada para as espécies de relevância no Brasil, Passiflora alata e Passiflora edulis. Propõe-se também o uso deste método para as espécies Maytenus aquifolium e Maytenus ilicifolia, uma vez que não há procedimento para a análise quantitativa de flavonoides destas espécies na literatura, e a monografia sobre M. ilicifolia da Farmacopéia Brasileira propõe apenas a dosagem de taninos totais (Farmacopéia Brasileira, 2002). Este trabalho também apresenta a comparação do método espectrométrico com os resultados obtidos na análise por CLAE dos flavonoides da espinheira-santa e do maracujá.

\section{MATERIAL E MÉTODOS}

\section{Material vegetal}

O material vegetal utilizado como referência para o desenvolvimento do método analítico foi gentilmente fornecido pela Dra. Ana Maria Soares Pereira (Unaerp, Ribeirão Preto-SP, Brasil), e foi obtido de espécimens originários de cultivo conforme boas práticas agrícolas na fazenda da Unaerp, Ribeirão Preto-SP, Brasil. As folhas de Maytenus aquifolium Mart. e Maytenus ilicifolia (Schrad.) Planch. (Celastraceae) foram coletadas em dezembro/2000, as folhas de Passiflora alata Curtis foram coletadas em março de 1999 e as de Passiflora edulis Sims
(Passifloraceae) em junho de 1998. As folhas foram secas em estufa a $40^{\circ} \mathrm{C}$, por dois dias, pulverizadas e tamizadas $(0,5-1,0 \mathrm{~mm})$ antes de serem armazenadas em frascos fechados, protegidos da luz.

\section{Reagentes e soluções}

Todos os reagentes utilizados foram de grau analítico: metanol (Mallinckrodt), etanol (Mallinckrodt e Merck), ácido fórmico 98-100\% (Merck), ácido acético glacial (Synth), ácido acético anidro (Merck), cloreto de alumínio (Merck), ácido bórico (Vetec) e ácido oxálico (Merck). Como padrão de flavonoide, utilizou-se rutina 95\% (Sigma). Utilizou-se água deionizada em sistema Milli-Q (Millipore).

\section{Extração do material vegetal e análise espectrométrica}

Procedimento modificado da Farmacopéia Francesa (F.F.)

Folhas pulverizadas $(0,3 \mathrm{~g})$ foram extraídas com etanol:água $60 \% \mathrm{v} / \mathrm{v}(20 \mathrm{~mL})$ sob refluxo $(60 \mathrm{~min})$. $\mathrm{O}$ extrato foi resfriado à temperatura ambiente e filtrado; o resíduo (material vegetal) foi re-extraído seguindo o mesmo procedimento. Ambos os extratos hidroetanólicos foram reunidos e o volume ajustado para $50,0 \mathrm{~mL}$ em balão volumétrico com etanol:água $60 \% \mathrm{v} / \mathrm{v}$, obtendo-se a solução de trabalho. Uma alíquota de $0,8 \mathrm{~mL}$ da solução de trabalho foi transferida para um balão volumétrico de $10 \mathrm{~mL}$ e o volume ajustado com metanol p.a. (Solução 1). Uma segunda alíquota de $0,8 \mathrm{~mL}$ da solução de trabalho foi transferida para um outro balão volumétrico de 10 $\mathrm{mL}$, adicionou-se $0,8 \mathrm{~mL}$ de solução $\mathrm{AlCl}_{3} 2 \% \mathrm{~m} / \mathrm{V}$ e o volume ajustado com metanol p.a. (Solução 2). Após 25 min da adição da solução de $\mathrm{AlCl}_{3}$, a absorvância foi medida em espectrofotômetro Hitachi U-2000 a $\lambda=413$ $\mathrm{nm}$ para os extatos de Maytenus e $\lambda=427 \mathrm{~nm}$ para os extratos de Passiflora, usando a Solução 1 como branco. O procedimento descrito para a preparação das Soluções 1 e 2 foi também seguido para obtenção da curva analítica, usando como padrão soluções de rutina em etanol:água $60 \% \mathrm{v} / \mathrm{v}$ nas concentrações de 37,$5 ; 75,0 ; 112,5 ; 150,0$ e $187,0 \mathrm{mg} . \mathrm{L}^{-1}$ para análise dos extratos de Maytenus e de 100,$0 ; 140,0 ; 180,0 ; 220,0$ e 260,0 mg.L.-1 para análise dos extratos de Passiflora.

\section{Procedimento modificado da Farmacopéia Européia (F.E.)}

Folhas pulverizadas $(0,2 \mathrm{~g})$ foram extraídas com etanol:água $60 \% \mathrm{v} / \mathrm{v}(40 \mathrm{~mL})$ sob refluxo $(30 \mathrm{~min})$. O extrato foi resfriado à temperatura ambiente e filtrado; o resíduo (material vegetal) foi re-extraído seguindo o mesmo procedimento. Ambos os extratos hidroetanólicos foram reunidos e o volume ajustado para $100,0 \mathrm{~mL}$ em balão 
volumétrico com etanol:água $60 \%$ v/v, obtendo-se a solução de trabalho. Uma alíquota de 5,0 mL da solução de trabalho foi seca em rotaevaporador e o resíduo foi dissolvido em $0,9 \mathrm{~mL}$ metanol $+9,1 \mathrm{~mL}$ ácido acético glacial e transferido para um balão volumétrico de $25,0 \mathrm{~mL}$; adicionou-se 10 $\mathrm{mL}$ de ácido fórmico e o volume foi completado com ácido acético anidro (Solução 1). Uma segunda alíquota de 5,0 $\mathrm{mL}$ da solução de trabalho foi seca em rotaevaporador e o resíduo dissolvido em $0,9 \mathrm{~mL}$ metanol $+9,1 \mathrm{~mL}$ ácido acético glacial. A mistura foi transferida para um balão volumétrico de $25 \mathrm{~mL}$, em seguida adicionou-se $10 \mathrm{~mL}$ de uma solução consistindo de $0,25 \mathrm{~g}$ de ácido bórico $+0,20 \mathrm{~g}$ de ácido oxálico, diluídos em ácido fórmico e completouse o volume com ácido acético anidro (Solução 2). Após 30 min da adição da solução de $\mathrm{AlCl}_{3}$, a absorvância foi medida em espectrofotômetro Hitachi U-2000 a $\lambda=419$ $\mathrm{nm}$ para os extratos de Maytenus e $\lambda=430 \mathrm{~nm}$ para os extratos de Passiflora, usando a Solução 1 como branco. O procedimento descrito para a preparação das Soluções 1 e 2 foi também seguido para obtenção da curva analítica, usando como padrão soluções de rutina em etanol:água $60 \% \mathrm{v} / \mathrm{v}$ nas concentrações de 15,$0 ; 30,0 ; 45,0 ; 60,0$ e 75,0 mg.L $\mathrm{L}^{-1}$ para análise dos extratos de Maytenus e de 5,0; 10,0; 20,0; 30,0 e 40,0 mg. $\mathrm{L}^{-1}$ para análise dos extratos de Passiflora.

\section{Validação}

As metodologias descritas foram validadas quanto à precisão (repetibilidade e precisão intermediária), exatidão, linearidade e sensibilidade, segundo os critérios propostos pelo $\mathrm{ICH}(\mathrm{ICH}, 1996)$. A precisão foi avaliada pela repetibilidade, na qual foram examinadas três amostras individuais em um único dia, e pela precisão intermediária, a qual foi determinada em três dias diferentes. A exatidão foi avaliada por ensaios de recuperação. $O$ teste de recuperação consiste na adição de quantidades conhecidas da substância padrão (rutina) à amostra analisada. Todas as análises foram realizadas em triplicata. A linearidade foi determinada para a curva analítica da rutina em cinco diferentes valores de concentração (descritos no item Extração do material vegetal e análise espectrométrica) e todas as análises foram realizadas em triplicata. A sensibilidade do método foi determinada através da inclinação da curva analítica (Ribani et al., 2004).

Os resultados obtidos por espectrometria no UV-Visível foram também comparados, usando o teste $t$ de Student (nível de confiança de 95\%) (Miller \& Miller, 2002), com os dados quantitativos obtidos por CLAE seguindo métodos descritos em trabalhos anteriores (Pereira et al., 2004; Diagone, 2003).

\section{RESULTADOS E DISCUSSÃO}

Os procedimentos originalmente descritos na Farmacopéia Francesa (Pharmacopée Française, 1992) e na Farmacopéia Européia (European Pharmacopoeia, 2000) necessitaram de algumas modificações para se obter melhores resultados. Uma alteração foi o comprimento de onda utilizado para medição da absorvância: para a análise de Passiflora incarnata L. (Passifloraceae) a literatura indica leitura em 394 nm (F.F.) ou em 401 nm (F.E.). Porém, após a reação de complexação, obteve-se os valores de $\lambda_{\text {máx }}=412 \mathrm{~nm}$ (F.F.) e $419 \mathrm{~nm}$ (F.E.) para os extratos de Maytenus (Celastraceae), enquanto que para os extratos de Passiflora obteve-se $\lambda_{\text {máx }}=427$ nm (F.F.) e $430 \mathrm{~nm}$ (F.E.). Assim, conforme descrito em Material e Métodos, optou-se pela leitura de absorvância nos máximos de absorção, que é o procedimento mais correto para análise quantitativa. Maytenus aquifolium Mart. e Maytenus ilicifolia (Schrad.) Planch. contém flavonoides $O$-glicosídeos e suas agliconas (Tiberti et al., 2007), enquanto que os flavonoides de Passiflora são predominantemente $C$-glicosilados (Pereira et al., 2005); estas diferenças estruturais justificam as diferenças de valores de $\lambda_{\text {máx }}$ observados entre as espécies.

Outra modificação dos métodos espectrométricos descrito nas farmacopéias foi a escolha da rutina como substância de referência para construção da curva analítica e cálculo do teor de flavonoides totais. A utilização da rutina é justificada por ser um flavonol diglicosilado (derivado de quercetina), cuja estrutura química é próxima dos flavonoides glicosilados presentes nas espécies estudadas. Outro motivo está relacionado ao seu menor custo, comparado à vitexina (padrão indicado nas Farmacopéias Francesa e Européia) e outros padrões de flavonoides.

Os parâmetros de validação são apresentados na Tabela 1. O parâmetro de linearidade foi obtido por meio da curva analítica do padrão rutina, e constatou-se que ambos os métodos são lineares, apresentando valores de coeficiente de correlação $\left(\mathrm{r}^{2}\right)$ próximo de um, mas o método da Farmacopéia Francesa apresentou maior linearidade e maior sensibilidade para as espécies de Maytenus e de Passiflora. Ambos os métodos também se apresentaram precisos, e tanto a repetibilidade quanto a precisão intermediária apresentaram coeficiente de variação $(\mathrm{CV})$ inferior a 5\%. Os valores de recuperação entre 70 e $120 \%$ são aceitáveis segundo os limites propostos (ICH, 1996).

Os resultados da análise dos flavonoides totais presentes nas amostras de referência das espécies de Maytenus e de Passiflora foram comparados com os resultados obtidos por CLAE na análise destas mesmas amostras (Tabela 2). Foi aplicado o teste $t$ de Student no nível de confiança de 95\%, para comparação estatística entre os resultados e encontraram-se diferenças significativas entre os resultados obtidos pelas duas metodologias (F.F. e F.E.), para as mesmas espécies. O teor de flavonoides pelo método da Farmacopéia Européia foi maior, e uma hipótese é que outros constituintes químicos dos extratos de Maytenus e de Passiflora poderiam sofrer reação de complexação com o ácido bórico; outra hipótese é que hidroxilas catecólicas em 3'e 4' não são a única posição complexada. Já na comparação entre o método espectrométrico e a análise 
por CLAE, a Farmacopéia Francesa apresentou resultados estatisticamente idênticos $(\mathrm{p}=0,05$; teste- $t)$ aos obtidos por CLAE.

Tabela 1. Curva analítica do padrão analítico rutina e parâmetros de validação das metodologias estudadas.

\begin{tabular}{|c|c|c|c|c|c|}
\hline Espécie & Método & $\begin{array}{c}\text { Equação da curva } \\
y=a+b x\end{array}$ & $\begin{array}{c}\text { Repetibilidade }^{\mathrm{a}} \\
\text { CV }(\%)\end{array}$ & $\begin{array}{c}\text { Precisão } \\
\text { Intermediária }^{a} \\
\text { CV }(\%)\end{array}$ & $\begin{array}{c}\text { Recuperação } \\
\text { CV }(\%)\end{array}$ \\
\hline M. aquifolium & $\begin{array}{c}\text { F.F. } \\
\text { (UV-Vis) }\end{array}$ & $\begin{array}{c}y=0,0209+0,02783 x \\
\left(r^{2}=0,9993\right)\end{array}$ & $13,76(1,80)$ & $14,25(4,10)$ & $71,0(2,2)$ \\
\hline M. ilicifolia & & & $14,62(2,40)$ & 14,$64 ;(4,10)$ & $74,0(4,2)$ \\
\hline M. aquifolium & $\begin{array}{c}\text { F.E. } \\
\text { (UV-Vis) }\end{array}$ & $\begin{array}{c}\mathrm{y}=0,0039+0,03697 \mathrm{x} \\
\left(\mathrm{r}^{2}=0,9987\right)\end{array}$ & $17,88(2,70)$ & $20,25(10,40)$ & $119,0(8,5)$ \\
\hline M. ilicifolia & & & $19,63(0,73)$ & $20,16(5,30)$ & $98,0(12,0)$ \\
\hline P. edulis & $\begin{array}{c}\text { F.F. } \\
\text { (UV-Vis) }\end{array}$ & $\begin{array}{c}y=-0,0123+28,3125 x \\
\left(r^{2}=0,9982\right)\end{array}$ & $32,49(3,18)$ & $32,51(0,95)$ & $78,2(2,1)$ \\
\hline P. alata & & & $14,44(4,14)$ & $14,20(1,49)$ & $75,7(3,0)$ \\
\hline P. edulis & $\begin{array}{c}\text { F.E. } \\
\text { (UV-Vis) }\end{array}$ & $\begin{array}{c}y=0,05687+21,6061 x \\
\left(r^{2}=0,9958\right)\end{array}$ & $56,78(1,42)$ & $56,16(0,95)$ & $115,7(7,3)$ \\
\hline P. alata & & & $27,43(11,11)$ & $26,78(2,61)$ & $100,6(8,5)$ \\
\hline
\end{tabular}

F.F.= Farmacopéia Francesa; F.E.= Farmacopéia Européia; CV (\%) - coeficiente de variação percentual da triplicata; ${ }^{a}$ Resultado expresso em rutina ( $\mathrm{mg}$ de flavonóide/g de planta seca)

Tabela 2. Teor de flavonoides totais expressos em rutina ( $\mathrm{mg}$ de flavonoide/g de planta seca), obtido por espectrometria no UVVisível e por CLAE-UV/DAD.

\begin{tabular}{|c|c|c|c|c|c|c|}
\hline \multirow{2}{*}{ Espécies } & \multirow{2}{*}{ Método } & \multicolumn{4}{|c|}{ Teor de flavonóides totais (TFT) } & \multirow{2}{*}{$\mathrm{CV}(\%)$} \\
\hline & & $\mathrm{TFT}_{1}$ & $\mathrm{TFT}_{2}$ & $\mathrm{TFT}_{3}$ & Média & \\
\hline \multirow[t]{3}{*}{ M. aquifolium } & F.F. (UV-Vis) & 13,76 & 14,90 & 14,10 & $14,25^{\mathrm{a}}$ & 4,1 \\
\hline & F.E. (UV-Vis) & 17,88 & 21,15 & 21,71 & 20,25 & 10,4 \\
\hline & CLAE $^{\mathrm{I}}$ & 13,56 & 13,51 & 12,81 & $13,30^{\mathrm{a}}$ & 2,6 \\
\hline \multirow[t]{3}{*}{ M. ilicifolia } & F.F. (UV-Vis) & 15,25 & 14,06 & 14,62 & $14,64^{\mathrm{b}}$ & 4,0 \\
\hline & F.E. (UV-Vis) & 19,45 & 21,40 & 19,63 & 20,16 & 5,3 \\
\hline & CLAE $^{\mathrm{I}}$ & 13,73 & 13,56 & 14,26 & $13,85^{\mathrm{b}}$ & 3,1 \\
\hline \multirow[t]{3}{*}{ P. edulis } & F.F. (UV-Vis) & 10,23 & 11,56 & 10,52 & $10,77^{\mathrm{c}}$ & 6,49 \\
\hline & *F.E. (UV-Vis) & 56,78 & 55,85 & 55,86 & 56,16 & 0,95 \\
\hline & CLAE $^{I I}$ & 11,12 & 11,07 & 10,97 & $11,05^{\mathrm{c}}$ & 0,69 \\
\hline \multirow[t]{3}{*}{ P. alata } & F.F. (UV-Vis) & 10,75 & 10,59 & 8,31 & $9,88^{\mathrm{d}}$ & 13,81 \\
\hline & *F.E. (UV-Vis) & 27,43 & 26,04 & 26,86 & 26,78 & 2,61 \\
\hline & CLAEII & 11,23 & 11,49 & 11,02 & $11,25^{\mathrm{d}}$ & 2,09 \\
\hline
\end{tabular}

F.F.= Farmacopéia Francesa; F.E.= Farmacopéia Européia; TFT- Teor de flavonóides totais; CV (\%) - coeficiente de variação percentual; a,b,c,d Resultados estatisticamente idênticos ( $\mathrm{p}=0,05$; teste- $t)$; * granulometria das plantas $<0,5 \mathrm{~mm}$; ${ }^{\mathrm{I} D a d o s}$ descritos na literatura (Diagone, 2003) e obtidos com as mesmas amostras analisadas por UV-Visível; " ${ }^{I I}$ Dados descritos na literatura (Pereira et al., 2004) e obtidos com as mesmas amostras analisadas por UV-Visível.

Com base nestes resultados e considerando que a espectrometria no UV-Visível apresenta a importante vantagem de ser uma técnica simples, rápida, de baixo custo e que utiliza menos quantidade de solvente em comparação à CLAE, conclui-se que o método espectrométrico modificado da Farmacopéia Francesa, conforme descrito neste trabalho, pode ser uma alternativa para a quantificação de flavonoides de folhas de Maytenus aquifolium Mart., Maytenus ilicifolia (Schrad.) Planch. (Celastraceae), Passiflora alata Curtis e Passiflora edulis Sims (Passifloraceae) em trabalhos que exigem a análise de grande número de amostras, tais como em ensaios agronômicos (estudos de melhoramento vegetal) ou no controle de qualidade de flavonoides em extratos vegetais.

\section{AGRADECIMENTOS}

FAPESP, CNPq e CAPES. 


\section{REFERÊNCIAS}

Balmé, F 1982. Plantas Medicinais. São Paulo: Hemus.

Carlini ELA 1988. Estudo da ação anti-úlcera gástrica de plantas brasileiras: Maytenus ilicifolia (espinheirasanta) e outras. Brasília: CEME/AFIP.

Carvalho ACB, Balbino EE, Maciel A, Perfeito JPS 2008. Situação do registro de medicamentos fitoterápicos no Brasil. Rev Bras Farmacogn 18: 314-319.

Diagone CA 2003. Desenvolvimento de métodos cromatográficos (HPLC/DAD e eletroforético CZE/DAD) para análise de flavonóides em "espinheira-santa" (Maytenus aquifolium Martius $e$ Maytenus ilicifolia Martius). São Carlos, 259p. Tese de Doutorado, Instituto de Química de São Carlos, Universidade de São Paulo.

European Pharmacopoeia 2000. 3. ed. Supplement Strasbourg: Council of Europe.

Farmacopéia Brasileira 2002. 4. ed. São Paulo: Atheneu. International Conference on Harmonization (ICH) 1996. Guideline Q2B-Validation of Analytical Procedures: Methodology. International Conference on Harmonization of Technical Requirements for Registration of Pharmaceuticals for Human Use. Genebra, Suíça.

Mariot MP, Barbieri RL 2007. Metabólitos secundários e propriedades medicinais da espinheira-santa (Maytenus illicifolia Mart. ex Reiss. e M. aquifolium Mart.). Rev Bras Plantas Med 9: 89-99.

Mercadante AZ, Britton G, Rodriguez-Amaya DB 1998. Carotenoids from yellow passion fruit (Passiflora edulis). J Agr Food Chem 46: 4102-4106.

Miller JN, Miller JC 2002. Estadística y Quimiometría para Química Analítica. Madrid: Pearson Educación S.A.

Oga S, Freitas PCD, Silva ACG, Hanada S 1984. Pharmacological trials of crude extract of Passiflora alata. Planta Med 50 : 303-306.

Pereira CAM, Vilegas JHY 2000. Constituintes químicos e farmacologia do gênero Passiflora com ênfase a $P$. alata Dryander, P. edulis Sims e P. incarnata L. Rev Bras Plantas Med 3: 1-12.

Pereira CAM, Yariwake JH, Lanças FM, Wauters JN, Tits M, Angenot L 2004. HPTLC densitometric determination of flavonoids from Passiflora alata, P. edulis, P. incarnata and $P$. caerulea and comparison with HPLC method. Phytochem Analysis 15: 241-248.

Pereira CAM, Yariwake JH, McCullagh M 2005. Distinction of the flavone C-glycoside pairs orientin/isoorientin and vitexin/isovitexin using LC-MS exact mass measurement and in-source CID. Phytochem Analysis 16: 295-301.

Pharmacopée Française 1992. 10. ed. Paris: Ministère de la Santé.

Ribani M, Bottoli CBG, Collins CH, Jardim ICSF, Melo LFC 2004. Validação em métodos cromatográficos e eletroforéticos. Quim Nova 27: 771-780.

Schefer MC 2004. Produção de espinheira-santa (Maytenus ilicifolia Mart. Ex Reiss) na região metropolitana de
Curitiba, Paraná, Brasil. In: Alexiades MN, Shanley $\mathrm{P}$ (org) Productos forestales, medios de subsistencia y conservación. Indonésia: Centro para la Investigación Forestal Internacional, p. 329-349.

Souza-Formigoni MLO, Oliveira MGM, Monteiro MG, Da Silveira NG, Braz S, Carlini EA 1991. Antiulcerogenic effects of two Maytenus species in laboratory animals. $J$ Ethnopharmacol 34: 21-27.

Tiberti LA, Yariwake JH, Ndjoko K, Hostettmann K 2007. Identification of flavonols in leaves of Maytenus ilicifolia and $M$. aquifolium (Celastraceae) by LC/UV/MS analysis. J Chromatogr B 846: 378-384.

Yariwake JH, Lanças FM, Cappelaro EA, Vasconcelos EC, Tiberti LA, Pereira AMS, Franca SC 2005. Variabilidade sazonal de constituintes químicos (triterpenos, flavonóides e polifenóis) das folhas de Maytenus aquifolium Mart (Celastraceae). Rev Bras Farmacogn 15: 162-168. 